\title{
PREDICTED EFFICIENCY OF SI WIRE ARRAY SOLAR CELLS
}

\author{
M. D. Kelzenberg, M. C. Putnam, D. B. Turner-Evans, N. S. Lewis, H. A. Atwater \\ California Institute of Technology, Pasadena, CA 91125
}

\begin{abstract}
Solar cells based on arrays of CVD-grown Si nano- or micro-wires have attracted interest as potentially low-cost alternatives to conventional wafer-based Si photovoltaics [1-6], and single-wire solar cells have been reported with efficiencies of up to $3.4 \%$ [7]. We recently presented device physics simulations which predicted efficiencies exceeding $17 \%$, based on experimentally observed diffusion lengths within our wires [8]. However, this model did not take into account the optical properties of a wire array device -- in particular the inherently low packing fraction of wires within CVD-grown wire arrays, which might limit their ability to fully absorb incident sunlight. For this reason, we have combined a device physics model of $\mathrm{Si}$ wire solar cells with FDTD simulations of light absorption within wire arrays to investigate the potential photovoltaic efficiency of this cell geometry. We have found that even a sparsely packed array $(14 \%)$ is expected to absorb moderate (66\%) amounts of abovebandgap solar energy, yielding a simulated photovoltaic efficiency of $14.5 \%$. Because the wire array comprises such a small volume of $\mathrm{Si}$, the observed absorption represents an effective optical concentration, which enables greater operating voltages than previously predicted for Si wire array solar cells.
\end{abstract}

\section{INTRODUCTION}

Arrays of vertical Si micro- or nanowires may offer a low-cost alternative to crystalline Si wafers for photovoltaic applications. This geometry is predicted to reduce the requirements of material purity, volume, and processing difficulty; while still retaining efficiencies competitive with planar multicrystalline Si solar cells [1]. Si wire arrays can be grown from potentially low-cost Si sources, such as chlorosilane, by the vapor-liquid-solid (VLS) chemical vapor deposition (CVD) technique $[9,10]$. Wire arrays for photovoltaic applications have been grown on low-cost substrates such as glass $[2,5,6]$, and the use of flexible substrates may someday enable inexpensive processing such as roll-to-roll sheet processing.

Recently, a wire array peel-off technique has been described, which not only yields a flexible array of crystalline Si wires for photovoltaic applications, but also allows the crystalline Si growth substrate to be re-used for the further production of arrays [11, 12]. This is accomplished by embedding the wire arrays within a transparent polymer (PDMS), then peeling the film in-tact from the growth substrate. An example polymerembedded wire array from our labs is shown in the inset of Figure 1. We now turn our attention to predicting the photovoltaic efficiency which might be expected if a solar cell were realized from such a wire array.

This envisioned solar cell structure for this study is depicted in Figure 1. The choice of the cell geometry was guided by two fundamental goals: that it absorb nearly all above-bandgap incident sunlight, and that it collect nearly all excited photocarriers while operating at a reasonable photovoltaic potential. The latter is ensured by the radial p-n junction within each wire, as illustrated by the red and blue regions in Figure 1. This radial junction geometry decouples length scale of light absorption from that of charge collection, much like the vertical multijunction [13] or parallel multijunction [14] geometries. The diameter of each wire provides a short minority carrier collection path; while its length provides a long photon absorption path, and enables near-unity internal quantum efficiency across the entire above-bandgap solar spectrum. This effect was first observed some time ago [15], and more recent modeling has shown that it is optimized when the wire diameter is on the order of the minority carrier diffusion length [1, 8]. Diffusion lengths ranging from 2 to $10 \mu \mathrm{m}$ have been observed within our wires $[8,16,17]$, thus, a wire diameter of $3 \mu \mathrm{m}$ was chosen for these theoretical

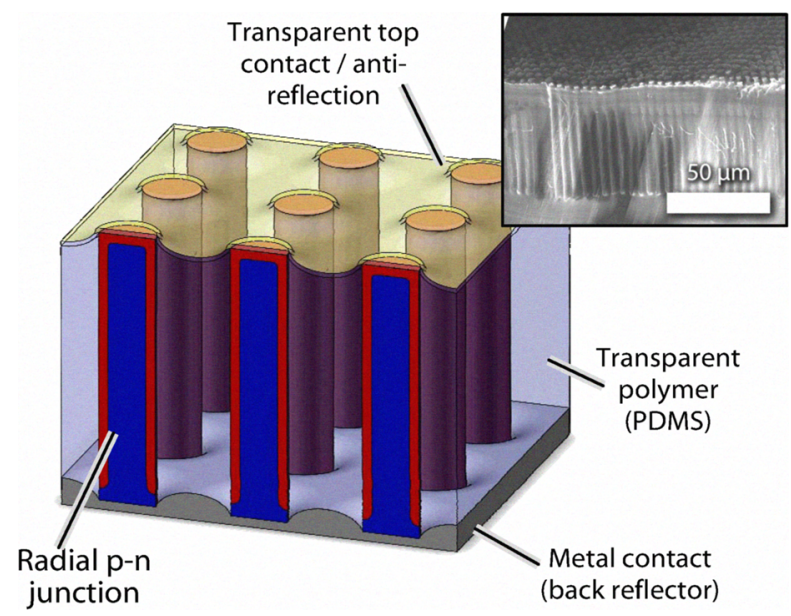

Fig. 1. Si wire array solar cell geometry modeled in this study. Inset: Scanning electron micrograph of polymerembedded Si wire array on which the envisioned cell geometry is based. 
studies. Although prior modeling [8] predicted that wire lengths of $\sim 100 \mu \mathrm{m}$ were optimal for photovoltaic energy conversion, computational resources limited the length of the wires in this study to $25 \mu \mathrm{m}$. Provisions for a transparent top contact and metallic bottom contact were included in the envisioned cell geometry, although their electrical properties (e.g. resistivity, contact barrier) were not considered.

The remaining design objective of the envisioned solar cell geometry is that it absorb nearly all abovebandgap incident sunlight. Several groups have studied $\mathrm{Si}$ nanowire array solar cells and their optical absorption properties [2, 5, 18, 19], reporting low reflectivity and enhanced absorption due the sub-wavelengths scale of the nanowires. However the wires of micron-scale diameter here are expected to interact with light much differently, so several simple light-trapping strategies were included in the modeled cell geometry. Firstly, the thickness of the top transparent contact (modeled as ITO) was selected to minimize the reflection of the top surface of the Si wires $(72 \mathrm{~nm})$. Secondly, the metallic back-side contact also served as a back-reflector. Finally, a slight curvature of the polymer film between wires was added to help scatter light within the wire array. Although this texturing was chosen arbitrarily, it mimics a similar curvature observed within the polymer-embedded wire arrays (see Figure 1 inset).

\section{OPTICAL SIMULATION OF SI WIRE ARRAYS}

Many optical effects are likely to be present within the wire array structures considered here, including scattering, diffraction, and waveguiding. Several groups have reported calculations of optical absorption in Si nanowire (sub-wavelength dimensions) arrays for photovoltaic applications, including models based on the transfer matrix method (TMM) [18], finite difference time domain (FDTD) simulations [20], or scattering theory [21]. Here, we have employed 3D FDTD simulations to calculate the absorption and resulting photogeneration profiles of the wire array structure, under monochromatic or synthesized AM 1.5G [22] illumination at normal incidence. Briefly, this method iteratively calculates the full-field $(\vec{E}, \vec{H})$ solutions to Maxwell's equations in the time domain, at each point on a $3 D$ simulation grid.

The simulation geometry is depicted in Figure 2(a). By placing periodic (Bloch) boundary conditions in the $x$ and $y$ directions, the simulations carried out within this unit cell model the periodic square-array wire structure from Figure 1. The back contact was modeled as a perfect electrical conductor for simplicity, although some losses would be expected at a real metallic reflector. The simulation volume was bounded above by a perfectly matched layer, allowing reflected light to escape the simulation volume. A plane wave source was placed several microns above the top surface of the wire array, and a reflection monitor located above this source monitored the total power reflected by the wire array $(R)$. In each simulation, the integrated Poynting power flow (a)
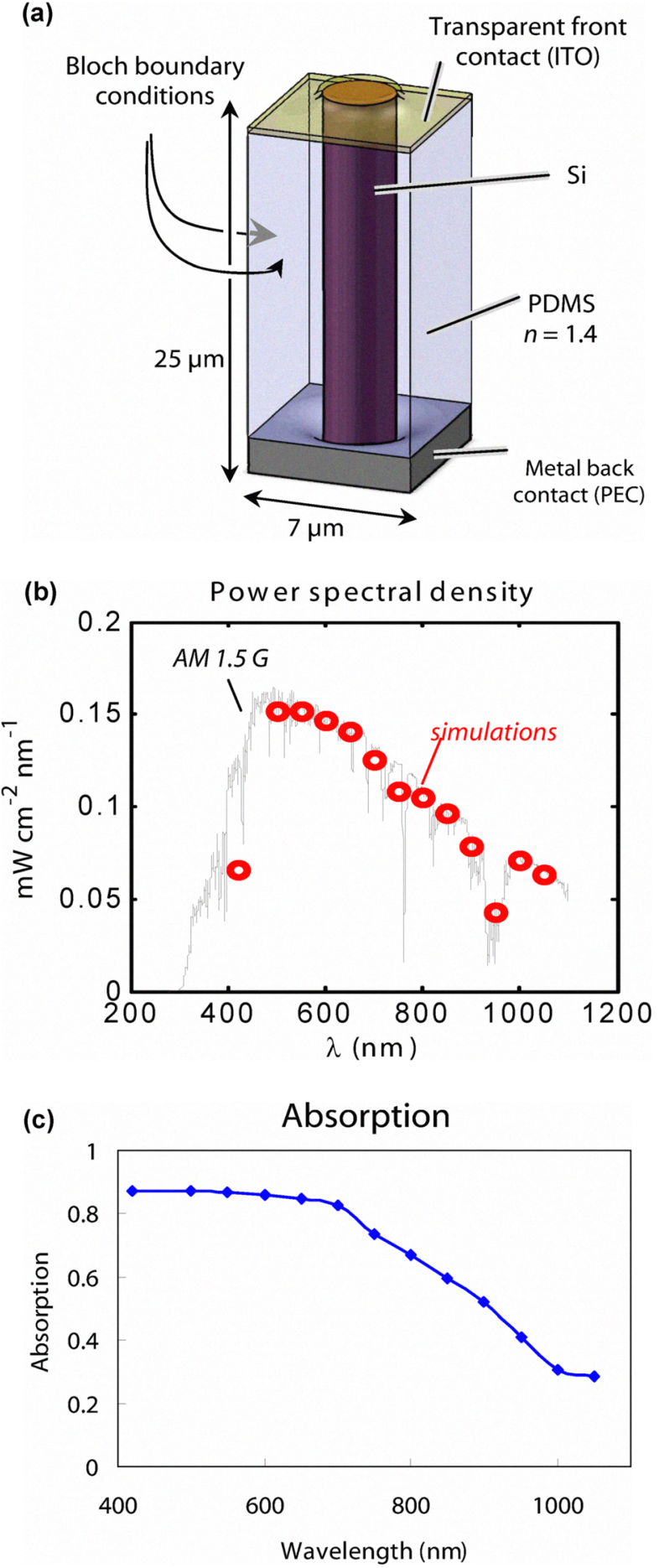

Figure 2. (a) Schematic of FDTD simulation configuration of Si wire array absorption. (b) Power spectral density plot of AM 1.5G spectrum, and the discrete simulations that were run to approximate it. (c) Absorption at each simulated wavelength, determined by 1 - R, where reflection was observed by a field monitor above the simulation volume. 
past the reflection monitor was recorded to calculate absorption, $A=1-R$.

The optical studies consisted of several singlewavelength simulations, as depicted in Figure 2(b). Computational resources limited the number of wavelengths that could be processed; thus, simulations were run at approximately $50 \mathrm{~nm}$ intervals throughout most of the region of interest to Si photovoltaics (280 $1100 \mathrm{~nm}$ ). The AM 1.5G spectrum was binned in this range, and the incident power in each simulation was weighted to match the respective photocurrent density originating within each band of sunlight. The total input power across all simulations totaled to $43.5 \mathrm{~mA} \mathrm{~cm}^{-2}$, the approximate upper limit of achievable photocurrent in a $\mathrm{Si}$ photovoltaic device by the principal of detailed balance. Partial spectral averaging $(\Delta \lambda \sim 25 \mathrm{~nm})$ was used to suppress coherence artifacts in the simulation results.

At each single-wavelength simulation, the materials' refractive and absorptive properties $(n, k)$ were determined based on published values for $\mathrm{Si}$ [23] and tabulated values for the ITO top contact [24]. In general, semiconductors can act both as absorbers and emitters of radiation, requiring device physics simulations to be coupled with optical simulations. However in this case the re-radiation of light from the Si wires could be safely ignored, due to the material's indirect bandgap and trap-dominated recombination. A fixed $n$ of 1.4 was used for the PDMS material. Simulations were performed on a full 3D grid using the software package FDTD Solutions (Lumerical, Inc.). Structures were meshed with no fewer than 20 grid points per media wavelength, and simulations were run until field intensities within the simulation volume had decayed to below $10^{-6}$ times their initial value.

The calculated absorption is plotted in Figure 2(c). Although the calculations indicate high $(>80 \%)$ absorption at visible wavelengths, this value decreases at infra-red wavelengths, yielding an overall AM1.5G-weighted absorption efficiency of $66 \%$. Although this is inadequate for highly-efficient photovoltaic applications, it would likely be improved by considering longer wire lengths or enhanced light-trapping schemes. Furthermore, it is important that future studies address the effects of incidence angle on absorption, particularly for non-tracking photovoltaic applications. This study made no attempt to optimize wire absorption, focusing instead on the implications of these results on the predicted photovoltaic efficiency of the envisioned wire array solar cell.

\section{DEVICE PHYSICS SIMULATIONS}

For this study, our previously reported device physics model [8] was modified to take into account the interaction of light with the Si wires. This model was implemented within the TCAD software suite (Synopsys, Inc.), using the Sentaurus device physics simulator (Version A-2008.09), which calculates the terminal $I-V$ behavior of a semiconductor device by simultaneously solving the electron and hole continuity equations and the Poisson equation on a simulation grid [25]. To simulate the behavior of the device under illumination, a non-zero photogeneration profile $\left(G_{\text {opt }}\right)$ is used as an input to the continuity equations. Previously, we employed a simple Beer's law absorption model to calculate the photogeneration profile within the Si wire device. In this study we used the output of the above FDTD simulations, processed as follows, as the input photogeneration profile for the device physics simulations.

The process of fundamental optical absorption in a semiconductor transfers energy from a propagating electromagnetic wave to excite a free electron-hole pair within the semiconductor; a transfer which is quantized by the photon energy, $E_{p h}=\hbar \omega$. Thus in the absence of other processes, the photogeneration rate can be determined from the divergence of Poynting vector $\vec{P}$. Under steady-state harmonic conditions this can be expressed in terms of the electric field intensity $\vec{E}$ and the imaginary part of the material's permittivity, $\varepsilon^{\prime \prime}$.

$$
G_{o p t}=\frac{1}{2 \hbar \omega} \operatorname{re}\{\vec{\nabla} \cdot \vec{P}\}=\frac{\varepsilon^{\prime \prime}|\vec{E}|^{2}}{2 \hbar}\left[\mathrm{cm}^{-3} \cdot \mathrm{s}^{-1}\right]
$$

In the 3D FDTD simulations described in the previous section, the steady-state electromagnetic field phasors ( $\vec{E}, \vec{H}$ ) at the simulation frequency were recorded at each simulation grid point, from which the optical generation profile, $G_{o p t}(x, y, z)$, was calculated using equation 1 .

The device physics model operated on a different grid and coordinate system than the FDTD simulations. The wire device was modeled on a $2 \mathrm{D}$ simulation grid under assumption of cylindrical symmetry in the third $(\theta)$

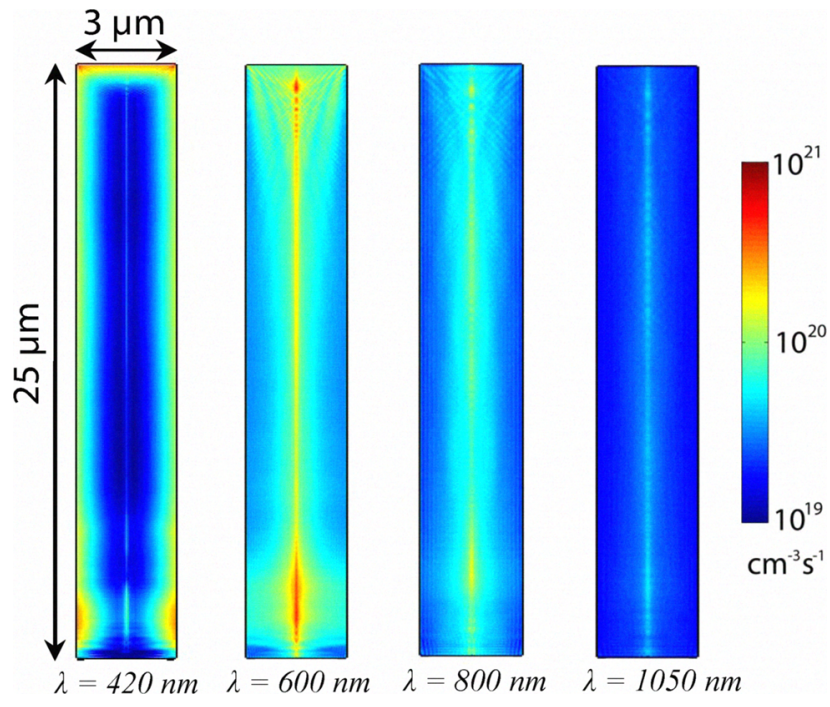

Fig. 3. Absorption profiles calculated by FDTD simulations. Each image plots the optical generation rate $G_{o p t}^{\prime}$ calculated by equation 2 , mirrored about $r=0$ to represent the vertical cross section through the center the wire. Four of the simulated wavelengths are represented here: 420 $\mathrm{nm}, 600 \mathrm{~nm}, 800 \mathrm{~nm}, 1050 \mathrm{~nm}$. Color map applies to all four panels. 
dimension. Although the 3D photogeneration profiles from FDTD were not strictly cylindrically symmetric, their variation as a function of the polar coordinate $(\theta)$ was minimal compared to that in the vertical $(z)$ or radial $(r)$ directions. Thus, device physics simulations were performed under the assumption of cylindrical symmetry, for which 3D optical generation profiles were flattened to a 2D cylindrical coordinates by numerical interpolation and integration:

$G_{o p t}^{\prime}(r, z)=\frac{\int_{0}^{2 \pi} r \cdot G_{o p t}(r \cos \theta, r \sin \theta, z) \cdot d \theta}{2 \pi r}\left[\mathrm{~cm}^{-3} \cdot \mathrm{s}^{-1}\right]$

This flattening procedure conserved the total photogeneration current within the device model; so that the resulting absorption, when integrated throughout the wire volume, agreed well with the absorption inferred from reflection during the FDTD simulations (Figure 2c). As a final step, the photogeneration profile was transferred to the non-uniformly spaced simulation grid of the device physics simulator. To prevent error in this re-sampling process, the profile was first interpolated onto a fine intermediate grid, and then averaged throughout the area corresponding to each simulation grid point.

The photogeneration profiles calculated at several wavelengths are shown in Figure 3 . These profiles have been flattened as described above, and mirrored about $r$ $=0$ for ease of viewing. The absorption profile for blue wavelengths (e.g. $420 \mathrm{~nm}$ ) is concentrated near the top and sides of the wire due to the short absorption length of $\mathrm{Si}$ at these photon energies. However, the generation rates for most of the solar spectrum (e.g. $600 \mathrm{~nm}$ and above) are elevated near the core, as a result of the waveguiding and focusing of light by the Si wires.

Figure 4(a) depicts the geometry and conditions of the device physics simulations. Unless otherwise specified, the default parameters for Si were used for all physical processes. A drift-diffusion model for carrier transport was employed, with doping-dependent mobility. Shockly-ReedHall (SRH), Auger, and surface recombination were considered. The SRH recombination lifetime was assumed to be constant throughout the device, chosen to yield a $10 \mu \mathrm{m}$ diffusion length in the wire's p-type $(B)$ core $\left(N_{A}=10^{18} \mathrm{~cm}^{-3}\right)$. A surface recombination velocity (SRV) of $1300 \mathrm{~cm} \cdot \mathrm{s}^{-1}$ was imposed at the surface of the wires. Both of these choices correspond to experimental observations within our wires [8]. The n-type $(P)$ emitter was assumed to have a Gaussian profile with a surface doping concentration of $5 \times 10^{18} \mathrm{~cm}^{-3}$, and a junction depth of $50 \mathrm{~nm}$. Finally, the contact boundary conditions were considered to be ideally ohmic, with minority carrier SRV of $10^{7} \mathrm{~cm} \cdot \mathrm{s}^{-1}$. The $I-V$ behavior of this device was simulated under each of the single-wavelength FDTD photogeneration profiles calculated above, as well as under the weighted, summed photogeneration profile representing AM 1.5G illumination. (a)

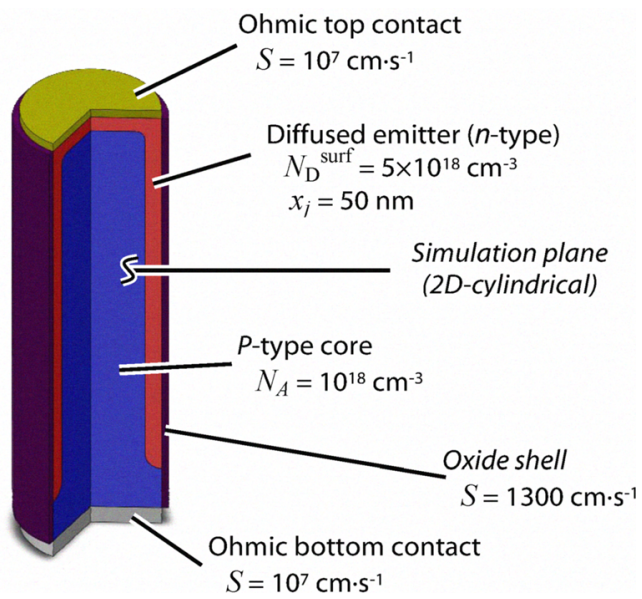

(b)
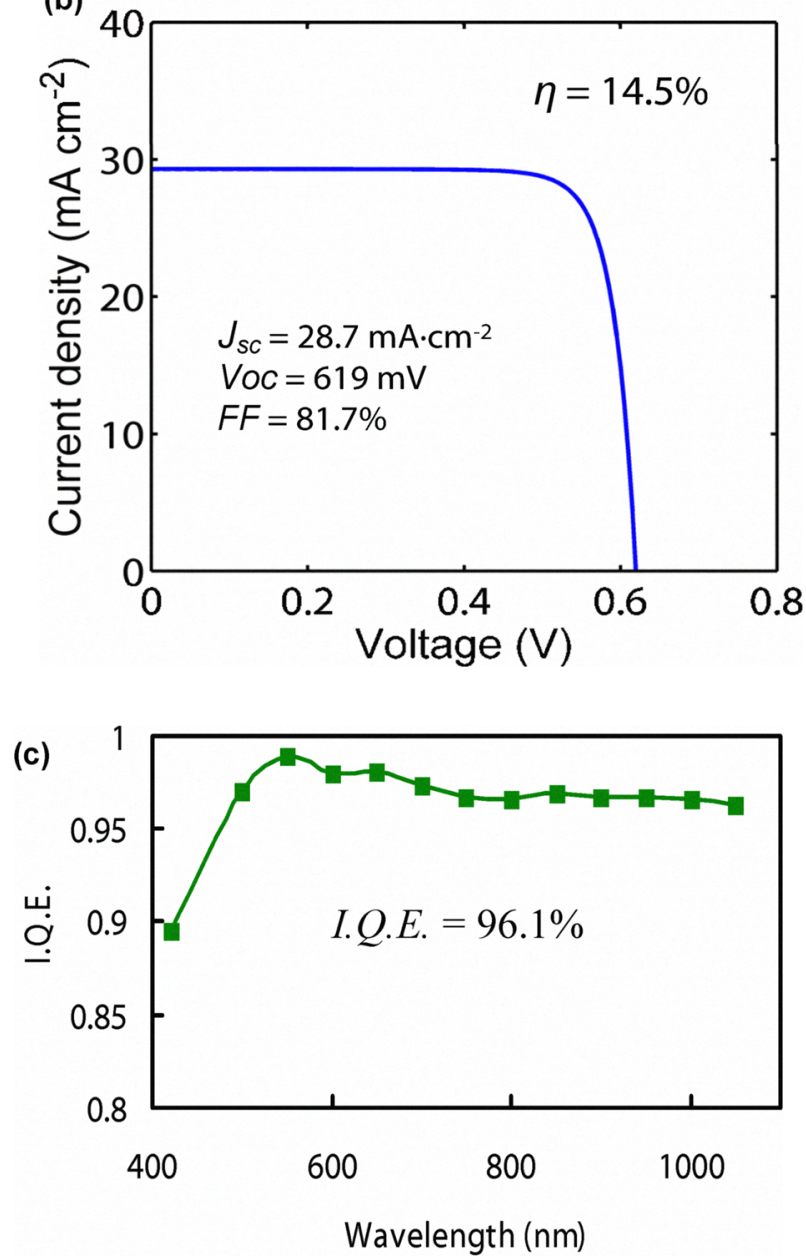

Fig. 4. (a) Schematic of device physics simulation, showing simulation plane and boundary conditions. (b) Simulated $J-V$ behavior of the wire array photovoltaic device (one unit cell) under synthesized AM1.5G illumination conditions. (c) Internal quantum efficiency simulated under each illumination wavelength, based on absorption data shown in figure 2(c). 
The simulated $J-V$ behavior of the wire array solar cell is shown in Figure 4(b). Unlike our prior modeling, which normalized current to the circular cross-sectional area of the wire only, the current here has been normalized to the entire $7 \mu \mathrm{m} \times 7 \mu \mathrm{m}$ unit cell of the wire array. Thus, this represents the actual $J-V$ behavior predicted for a macroscopic wire array solar cell. The simulated efficiency of this cell $(14.5 \%)$ is lower than that reported by our prior model (17.5\%), which did not consider the arrays' optical properties. This lower efficiency is due to a low short circuit current density $\left(29 \mathrm{~mA} \cdot \mathrm{cm}^{-2}\right)$, which was limited here by the $66 \%$ optical absorption of the wire array. Interestingly, however, the open-circuit voltage (620 $\mathrm{mV}$ ) is notably higher than the prior model predicted for identical wire dimensions, lifetime, and SRV (580 mV). This is due to effective optical concentration, since the $66 \%$ absorption occurs within wires that occupy only $14 \%$ of the illuminated area of the wire array. Normalized to the area of the wires only, the short circuit current density in this simulation is $200 \mathrm{~mA} \mathrm{~cm}$, indicating an effective concentration of nearly $5 \mathrm{x}$. Efficient photovoltaic applications will require absorption closer to $100 \%$, which might be achievable with enhanced light trapping (although this is beyond the scope of this study.) However, if near-unity absorption were achievable at a low wire packing fraction, the optical concentration effect observed here could enable cell efficiencies which even exceed that predicted by prior modeling.

The cell's internal quantum efficiency (IQE) was also calculated at each single-wavelength absorption profile, plotted in Figure 4(c). This is the ratio of collected carriers (from device physics simulations) to absorbed photons (from FDTD simulations, Figure 2c). As expected for the radial junction geometry, the IQE was near unity across all simulated wavelengths, yielding a weighted AM 1.5G average IQE of $96 \%$. The slight decrease in IQE at $\lambda=$ $420 \mathrm{~nm}$ is likely due to surface recombination, particularly the top of the wire where $S_{\text {contact }}=10^{7} \mathrm{~cm} \cdot \mathrm{s}^{-1}$. As noted in Figure 3 , absorption at this wavelength is concentrated nearer the surfaces (particularly the top) of the wire, and thus collection is more sensitive to surface recombination. However, this decrease in I.Q.E. is minimal even at 420 $\mathrm{nm}$. We have previously commented on this cell's insensitivity to surface recombination due to the radial junction geometry [8].

These results can interpreted in another way by comparing the radial junction to similar junction geometries such as the vertical multijunction [13] or parallel multijunction [14] solar cells. Common to these designs is a non-planar, interdigitated $\mathrm{p}-\mathrm{n}$ junction whose aspect ratio is chosen to accommodate the deficiency between diffusion length and absorption length within the semiconductor. This enables near-unity IQE despite arbitrarily low diffusion lengths, as observed here. But when realized within a planar absorber (e.g. a Si wafer) the total junction area of these geometries is inevitably scaled by this aspect ratio. It has been suggested that any theoretical increase in short-circuit current density would be offset by a greater decrease in operating voltage for multijunction geometries within planar absorbers [26].
However, this may not be the case for the wire array geometry. Consider the simple expression for $V_{o c}$ within a solar cell, as a function of its illuminated area, $A_{\text {ilum }}$ and junction area, $A_{j c n}$ :

$$
V_{o c}=\frac{k T}{q} \ln \frac{A_{\text {illum }} \cdot J_{\text {light }}}{A_{j c n} \cdot J_{\text {dark }}}
$$

In prior modeling we considered a single, vertical Si wire solar cell; illuminating and normalizing by the top (circular) surface area of the wire only. For the wire dimensions in the present study, these assumptions yield a predicted $V_{o c}$ reduction of $\sim 90 \mathrm{mV}$ in equation 3 , due to a $\sim 33 x$ junction area scaling. However in modeling the wire array solar cell, we also considered the sunlight incident upon the areas between the wires, much of which is eventually absorbed by the wires. Considering the $14 \%$ packing fraction of the wire array in this study, the predicted $V_{o c}$ reduction in equation 3 is only $\sim 40 \mathrm{mV}$. Assuming absorption could remain high, wire array solar cells might even be made at even lower packing fractions, which would further reduce the junction area to illumination area ratio. Thus, wire array solar cells are not necessarily bound by the open-circuit voltage loss inherent to highaspect-ratio junctions within planar absorbers.

\section{CONCLUSIONS}

Solar cells based on arrays of $\mathrm{Si}$ wires are a promising approach to low-cost, efficient photovoltaic devices. We have modeled the optical absorption of a Si wire array using FDTD, calculating that it would absorb $66 \%$ of above-bandgap sunlight. The resulting photogeneration profiles were used in a device physics model, in which the envisioned Si wire array solar cell exhibited $14.5 \%$ efficiency; broadband, near-unity IQE; and $620 \mathrm{mV}$ $V_{o c}$ despite a $10 \mu \mathrm{m}$ diffusion length. Although further work is required to increase the predicted absorption of $\mathrm{Si}$ wire arrays, the present observation implies an effective optical concentration of $\sim 5 x$. By combining high absorption, low packing fraction, and the radial junction geometry, Si wire array solar cells have the potential to reach efficiencies competitive with traditional crystalline $\mathrm{Si}$ solar cells.

\section{ACKNOWLEDGEMENTS}

The authors would like to thank Melissa Archer, Shannon Boettcher, Stanley Burgos, Vivian Ferry, Michael Filler, Brendan Kayes, Jim Maiolo, Stephen Maldonado, Evan and April Neidholdt, Domenico Pacifici, Jan Petykiewicz, Katherine Plass, Jessica Roberts, Josh Spurgeon, Luke Sweatlock, and Emily Warren.

This work was supported by BP; the Department of Energy, Office of Basic Energy Sciences; and the Center for Science and Engineering of Materials, an NSF Materials Research Science and Engineering Center at Caltech. 


\section{REFERENCES}

[1] B. M. Kayes, H. A. Atwater, and N. S. Lewis, "Comparison of the device physics principles of planar and radial p-n junction nanorod solar cells," Joumal of Applied Physics, 97, pp. 114302-11, 2005.

[2] L. Tsakalakos, J. Balch, J. Fronheiser, B. A. Korevaar, O. Sulima, and J. Rand, "Silicon nanowire solar cells," Applied Physics Letters, 91, pp. 233117-3, 2007.

[3] J. R. I. Maiolo, B. M. Kayes, M. A. Filler, M. C. Putnam, M. D. Kelzenberg, H. A. Atwater, and N. S. Lewis, "High Aspect Ratio Silicon Wire Array Photoelectrochemical Cells," Joumal of the American Chemical Society, 129, pp. 12346-12347, 2007.

[4] A. P. Goodey, S. M. Eichfeld, K.-K. Lew, J. M. Redwing, and T. E. Mallouk, "Silicon Nanowire Array Photoelectrochemical Cells," Journal of the American Chemical Society, 129, pp. 12344-12345, 2007.

[5] T. Stelzner, M. Pietsch, G. Andra, F. Falk, E Ose, and S. Christiansen, "Silicon nanowire-based solar cells," Nanotechnology, p. 295203, 2008.

[6] V. Sivakov, G. Andrä, A. Gawlik, A. Berger, J. Plentz, F. Falk, and S. H. Christiansen, "Silicon NanowireBased Solar Cells on Glass: Synthesis, Optical Properties, and Cell Parameters," Nano Letters, 9, pp. 1549-1554, 2009.

[7] B. Tian, X. Zheng, T. J. Kempa, Y. Fang, N. Yu G. Yu, J. Huang, and C. M. Lieber, "Coaxial silicon nanowires as solar cells and nanoelectronic power sources," Nature, 449, pp. 885-889, 2007.

[8] M. D. Kelzenberg, M. A. Filler, B. M. Kayes, M. C. Putnam, D. B. Turner-Evans, N. S. Lewis, and H. A. Atwater, "Single-nanowire Si solar cells," in Proceedings of the 33rd IEEE PVSC, 2008.

[9] R. S. Wagner and W. C. Ellis, "Vapor-Liquid-Solid Mechanism of Single Crystal Growth," Applied Physics Letters, 4, pp. 89-90, 1964.

[10] B. M. Kayes, M. A. Filler, M. C. Putnam, M. D. Kelzenberg, N. S. Lewis, and H. A. Atwater, "Growth of vertically aligned Si wire arrays over large areas $\left(>1 \mathrm{~cm}^{2}\right)$ with Au and Cu catalysts," Applied Physics Letters, 91, pp. 103110-3, 2007.

[11] K. E. Plass, M. A. Filler, J. M. Spurgeon, B. M Kayes, S. Maldonado, B. Brunschwig, H. Atwater, and N S. Lewis, "Flexible Polymer-Embedded Si Wire Arrays," Advanced Materials, 21, pp. 325-328, Jan 2009.

[12] J. M. Spurgeon, K. E. Plass, B. M. Kayes, B. S. Brunschwig, H. Atwater, and N. Lewis, "Repeated epitaxial growth and transfer of arrays of patterned, vertically aligned, crystalline Si wires from a single Si substrate,"
Applied Physics Letters, 93, pp. 032112-3, 2008.

[13] W. P. Rahilly, "Vertical multijunction solar cells," in Record of the $19^{\text {th }}$ IEEE PVSC, 1972, pp. 44-52.

[14] M. A. Green and S. R. Wenham, "NOVEL PARALLEL MULTIJUNCTION SOLAR-CELL," Applied Physics Letters, 65, pp. 2907-2909, Dec 1994.

[15] A. A. Shchetinin, A. I. Drozhzhin, N. K. Sedykh, and E. P. Novokreshchenova, "Photoconverters bbased on silicon-crystal whiskers," Measurement Techniques, 41, pp. 502-204, 1978.

[16] M. D. Kelzenberg, D. B. Turner-Evans, B. M. Kayes, M. A. Filler, M. C. Putnam, N. S. Lewis, and H. A. Atwater, "Photovoltaic Measurements in Single-Nanowire Silicon Solar Cells," Nano Lett., 8, pp. 710-714, 2008. C. P. Morgan, In preparation, 2008.

[18] L. Hu and G. Chen, "Analysis of Optical Absorption in Silicon Nanowire Arrays for Photovoltaic Applications," Nano Lett., 7, pp. 3249-3252, 2007.

[19] J. Zhu, Z. Yu, G. F. Burkhard, C.-M. Hsu, S. T. Connor, Y. Xu, Q. Wang, M. McGehee, S. Fan, and Y. Cui, "Optical Absorption Enhancement in Amorphous Silicon Nanowire and Nanocone Arrays," Nano Letters, 9 , pp. 279-282, 2009.

[20] L. Tsakalakos, J. Balch, J. Fronheiser, M.-Y. Shih, S. F. LeBoeuf, M. Pietrzykowski, P. J. Codella, B. A. Korevaar, O. V. Sulima, J. Rand, A. Davuluru, and U. Rapol, "Strong broadband optical absorption in silicon nanowire films," Journal of Nanophotonics, 1, pp. $013552-$ 10, 2007.

[21] R. A. Street, P. Qi, R. Lujan, and W. S. Wong, "Reflectivity of disordered silicon nanowires," Applied Physics Letters, 93, pp. 163109-3, 2008.

[22] ASTM, "ASTM G173 - 09e1 Standard Tables for Reference Solar Spectral Irradiances: Direct Normal and Hemispherical on $37^{\circ}$ Tilted Surface", 2003.

[23] D. E. Aspnes, "Optical Properties of Si," in Properties of crystalline silicon, R. Hull, Ed. London UK: INSPEC, IEE, 1999, p. 677.

[24] J. A. Wollam Co, Inc. "WWASE32 Spectroscopic Ellipsometry Software," 2006.

[25] Synopsys_Inc., "Sentaurus Device User Guide," Version A-2007.12 ed, 2007.

[26] M. J. Stocks, A. Cuevas, A. W. Blakers, "Theoretical comparison of conventional and multilayer thin silicon solar cells," Progress in Photovoltaics: Research and Applications, 4, pp. 35-54, 1996. 